\author{
Military Technical College \\ Kobry El-Kobbah, \\ Cairo, Egypt.
}

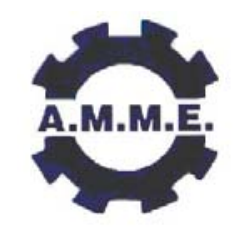

\title{
A MALAYSIAN PORTABLE THERMOACOUSTIC REFRIGERATOR SYSTEM
}

\author{
MOHD-GHAZALI ${ }^{1}$ N., CHENG C.F. ${ }^{2}$ and NURUDIN S. ${ }^{3}$
}

\begin{abstract}
The discovery that thermoacoustic phenomena can generate cooling for refrigeration has escalated research into thermoacoustic heat engine and refrigeration systems since the late twentieth century in developed countries. The simplicity of the thermoacoustic systems and its environmentally friendly operations has encouraged researchers in Malaysia to develop similar systems for specific purposes. A portable thermoacoustic refrigerator prototype using pure Helium as the working fluid was designed, fabricated and tested for thermoacoustic cooling effects. Based on the standing wave system with sinusoidal resonator geometry, a temperature drop was obtained repeatedly and consistently with cooling effects lower than the ambient when operating only at 1 atmospheric pressure. This paper reports the theory behind the design and fabrication procedure with initial temperature drop of $20 \mathrm{~K}$ obtained from the first thermoacoustic refrigerator in Malaysia. Investigations into different geometry stack and the complete refrigerator system are underway to study the optimum performance that can be obtained.
\end{abstract}

\section{KEY WORDS}

Thermoacoustics refrigerator, design, portable

1,3 Faculty of Mechanical Engineering, University Teknologi Malaysia, Malaysia FAX: 07-5566159, E-mail: normah@fkm.utm.my.

2 Curtin University of Technology Sarawak Campus, Sarawak, Malaysia. 


\section{INTRODUCTION}

Increasing awareness on sustainability of energy resources since the Brundtland Report [1] has spurred much research into alternative energy technologies across the globe. The Malaysian government, without exception, through government research bodies and public universities has continued its encouragement towards the exploration of sustainable production, processing, and utilization of its resources. Thermoacoustics technology, currently viewed as a potential replacement for the conventional refrigeration systems is one of the research area being pursued. With almost no moving parts and no hazardous refrigerants, a thermoacoustic cooling system may indeed be the answer to the ecological threat posed by the production of harmful refrigerants that is related to global warming and the thinning of the ozone layer.

Thermoacoustics theory is a field associated with either a temperature gradient induced by oscillations, or oscillations induced by a temperature gradient. The former is an everyday event with negligible temperature gradients produced by sound waves. The practical usefulness of the phenomenon, however, has only been realized in the late eighties with the first cryocooler probably made by Hofler in 1985 [2]. The last twenty years have seen fast developments in research into the thermoacoustics effects and systems. Theoretical and experimental works are many in developed countries with numerical studies slowly catching up due much to the nonlinearities and multidimensional effects involved. NASA and the Los Alamos National Laboratory (LANL) USA had successfully developed a high efficiency power source based on thermoacoustic technology, while Ben and Jerry in collaboration with Penn State University in USA developed an ice cream refrigerator with 120 Watt power $[3,4]$. Universiti Teknologi Malaysia (UTM) through research grants from the Ministry of Science and Technology (MOSTI) has been involved in thermoacoustic research since 2002. Theoretical, numerical, and experimental studies are being pursued. This paper discussed the portable thermoacoustic system designed, fabricated, and tested at UTM.

\section{DESIGN PROCEDURE}

Numerous efforts have been made to improve the design of thermoacoustic refrigerators through optimization procedures, particularly at the stack geometry, working fluids, thermal insulation, duct and other parameters. This study design requirements followed those of Wetzel and Herman [5], and Tijani [6]. A refrigerator for a temperature difference of $35 \mathrm{~K}$ with operating pressure of $10 \mathrm{bar}$ with a frequency of $400 \mathrm{~Hz}$ was considered. Helium is used as the working gas because it has the highest sound velocity and thermal conductivity among the noble gases and is easily available locally. A parallel research in numerical study has shown that mixtures of the noble gases with a high viscosity produces a higher inertial force which results in greater thermoacoustic effects [7], as been proven theoretically, and this type of working gas will be considered once the current study has achieved the designed outputs.

The material Mylar, normally chosen by researchers for their practical thermoacoustic systems was not obtainable, thus a Low Density Polyethylene (LDPE) was used. This material has a thermal conductivity of $0.33 \mathrm{~W} / \mathrm{mK}$ and other which are comparable to that of Mylar [8]. Parallel stack geometry being one of the best stack geometry, was 
designed and fabricated. Thermoacoustic effect occurs within a thermal penetration depth of $\delta \mathrm{k}$ which is defined by

$$
\delta_{k}=\sqrt{\frac{\kappa_{s}}{\omega}}
$$

with $\kappa_{s}$ being the thermal diffusivity and $\omega$ the frequency. Using the thermophysical properties of Helium at $300 \mathrm{~K}, \delta_{k}=0.1 \mathrm{~mm}$ and $\delta_{v}=0.08 \mathrm{~mm}$. The plate separation for the stack is $0.35 \mathrm{~mm}$ which is 3.5 times the thermal boundary layer. $\delta_{v}$ is the viscous penetration depth, the distance over which momentum can diffuse in a time $\frac{1}{\pi f}$ given by

$$
\delta_{v}=\sqrt{\frac{2 \mu}{\rho \omega}} .
$$

To avoid disturbance to the acoustic field, Wheatley et. al. [9] recommended a stack spacing of between $2 \delta_{k}$ to $4 \delta_{k}$. Figure 1 shows the LDPE stack fabricated.

The stack was to be placed about one-eighth of the distance of the resonator away from the acoustic driver [10]. As in any operating refrigerator system, heat exchangers are needed to transfer the energy of the thermoacoustic cooling process. Unlike the conventional heat exchanger design calculations, little is known about heat transfer in thermoacoustic system. The heat exchanger designed is the plate type made of copper for effective gas-solid heat transfer area. Figure 2 shows the heat exchanger fabricated at $0.5 \mathrm{~mm}$ in thickness for the cold side.

The resonator tube was designed and fabricated such that a quarter-wavelength standing wave fits across the length. Hofler [2] had shown that the resonator can be optimized by reducing the diameter of the tube on the right side of the stack. The resonator tube, made from aluminum, will then consist of 2 parts, the larger part comprising the stack and a smaller tube after. A cone-shaped buffer end used by Tijani to simulate an open end and thus reduce thermal losses was duplicated here [6]. The tube body, however, had been designed following that of Xiaofan et. al. [11], with a sinusoidal shape section before joining the buffer zone component. This varying crosssectional axisymmetric tube allows for a shock-free acoustic pressure conditions. Figure 3 shows the final shape of the resonator tube designed and fabricated at UTM.

The whole tube was designed such that it can be taken apart to accommodate different stack module (geometry and material) for research purposes as shown here in threedimension in Figure 4. The final total length of the resonator system inclusive of the driver housing is $807.59 \mathrm{~cm}$.

\section{EXPERIMENTAL SET-UP}

A schematic representation of the set-up is shown in Figure 5. Helium gas is supplied into the resonator tube at the required operating pressure. Although the resonator tube 
was fabricated to withstand up to 10 bar operating pressure, this research is looking at the performance curve from the lower end. In this report, results of experiments completed at the lower end of the operating pressure are shown. K-type thermocouples are used to measure the temperatures.

\section{RESULTS AND DISCUSSIONS}

In the initial experiment, when the system was injected with a $400 \mathrm{~Hz}$ (designed) acoustic waves from the function generator, the temperature difference recorded was too small. It is believed that with the driver housing included, the operating frequency may have changed slightly from the designed frequency. A series of experiments were done to identify the optimized operating frequency, Figure 6 shows the results of these experiments for various other frequencies attempted. Note that the highest temperature difference was actually attained at $420 \mathrm{~Hz}$. The possibility of fine-tuning the operating frequency could not be done due to unavailability of the necessary equipments. Although the temperature difference obtained is very much away from the designed temperature difference, Figure 7 shows significant thermoacoustic cooling effects. Unlike the Tijani's system [6], the system here was not placed in a vacuum and thus some effects from the humid environment may have occurred. The temperature at the cold end heat exchanger is lower than that of the ambient. The result here was obtained at 0.5 bar operating pressure and not at 1 bar in order to obtain a better drive ratio for the same pressure pulse, The drive ratio is the ratio of input pressure to the mean pressure. Note that the designed temperature drop of $35 \mathrm{~K}$ is actually expected at the operating pressure of $10 \mathrm{bar}$. The resonator was built to withstand this pressure and the particular experiment under this operating condition will be tested soon.

Experiments are being planned for higher operating pressures with higher drive ratios. The higher the drive ratio, the greater will be the thermoacoustic effects as reported by previous works. Various stack geometry is also being investigated given the constraints faced on the availability of local materials and technology.

\section{CONCLUSION}

The first portable thermoacoustic refrigeration system in Malaysia has been completed and experimental results showed a 10-degree temperature difference across the cold and hot heat exchangers, promising a potential for specific application. However, more tests are required to identify the capability of the system to achieve the designed cooling and work is currently underway to use better measuring equipments. It is unfortunate that budget limitations on equipment cost have imposed constraints on further development of the system as expected by the completed preliminary (numerical) studies.

\section{ACKNOWLEDGEMENT}

The authors would like to thank UTM and MOSTI for the funding to do this research. 


\section{REFERENCES}

[1] Brundtland report, UN General assembly document $A / 42 / 427$ available at http://en.wikisource.org/wiki/Brundtland-Report

[2] Hofler, T.J., Thermoacoustic Refrigerator Design and Performance, Ph.D. dissertation, University of California at San Diego, (1986).

[3] Garret, S.L., Adeff, J.A., and Hofler, T.J., Thermoacoustic Refrigerator for Space Applications, Journal of Thermophysics and Heat tRansfer, 7(4) (OctDec 1993).

[4] http://www.benjerry.com/our_company/sounds_cool/

[5] Wetzel M. and Herman C., Design Optimization of Thermoacoustic Refrigerators, Int J. Refrig. Vol. 20, No. 1, pp. 3 21, 1997

[6] Tijani, M.E.H., Loudspeaker-Driven Thermo-Acoustic Refrigeration, Ph.D. dissertation University of Eindhoven (2001).

[7] Cheng, C.F., and Mohd Ghazali, N., $5^{\text {th }}$ International Conference on Heat Transfer, Fluid Mechanics and Thermodynamics, Sun City, S. Africa, CC1 (2007).

[8] http://www.goodfellow.com/csp/active/STATIC/Polyethylene_Low_DEnsity.HTML. LDPE

[9] Wheatley, J.C., Hofler, T., Swift G.W., and Migliori A., Understanding Some Simple phenomena in Thermoacoustics with Applications to Acoustical Heat Engines, Am. J. Phys. 53, 147 (1985).

[10] Atchley, A.A., Hofler, T.J., Muzzerall, M.L., Kite, M.D., and Ao, C, Acoustically Generated Temperature Gradient in Short Plates, J. Acoust. Soc. Am. 88(1), July (1990).

[11] Xiaofan, L., Finkbeiner, J, Raman, G., Daniel, C., and Steinetz, B.M., Optimized Shapes of Oscillating Resonators for Generating High-Aplitude Pressure Waves, J. Acoust. Soc. Am. 116 (5) November (2004).

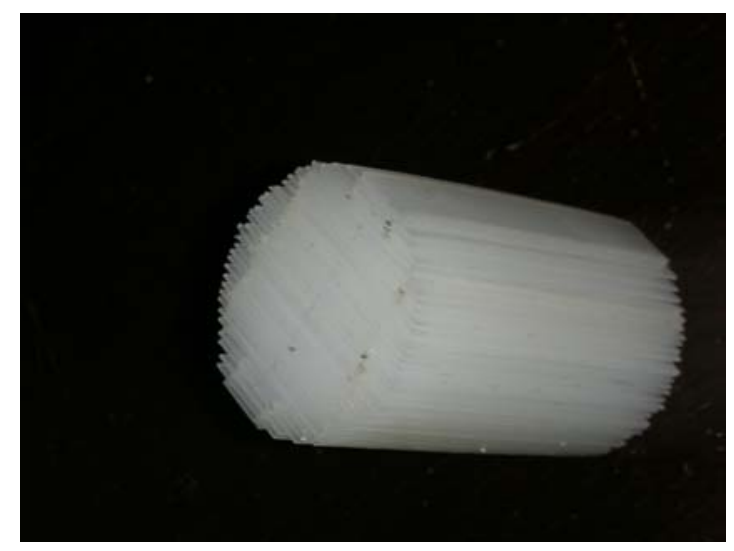

Fig. 1. The fabricated stack. 


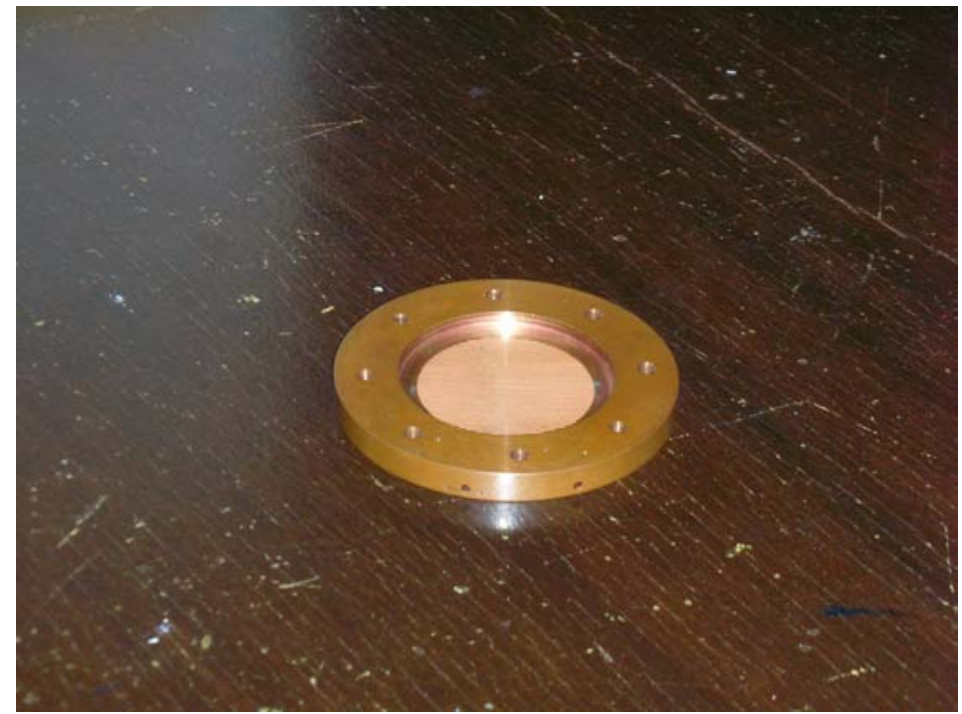

Fig. 2. The fabricated heat exchanger.

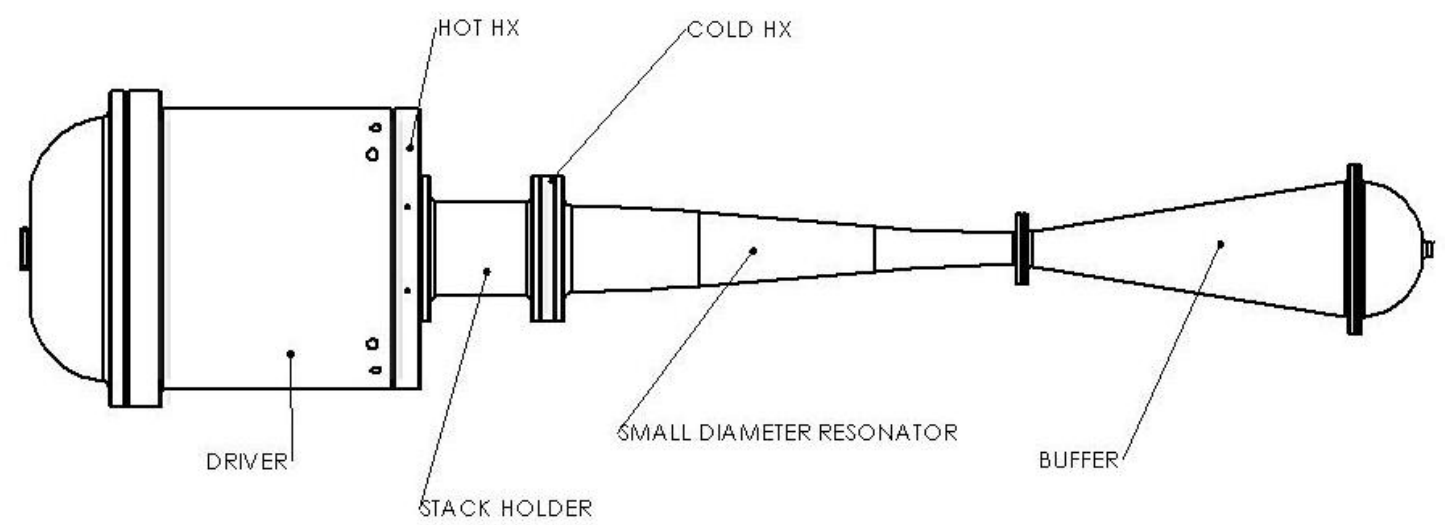

Fig. 3: A schematic of the UTM thermoacoustic system.

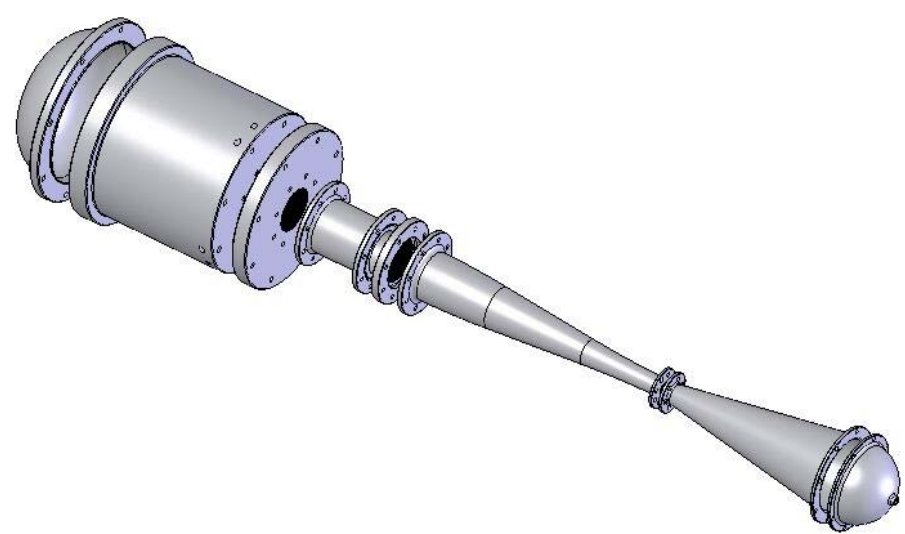

Fig. 4: 3D view of thermoacoustic system. 


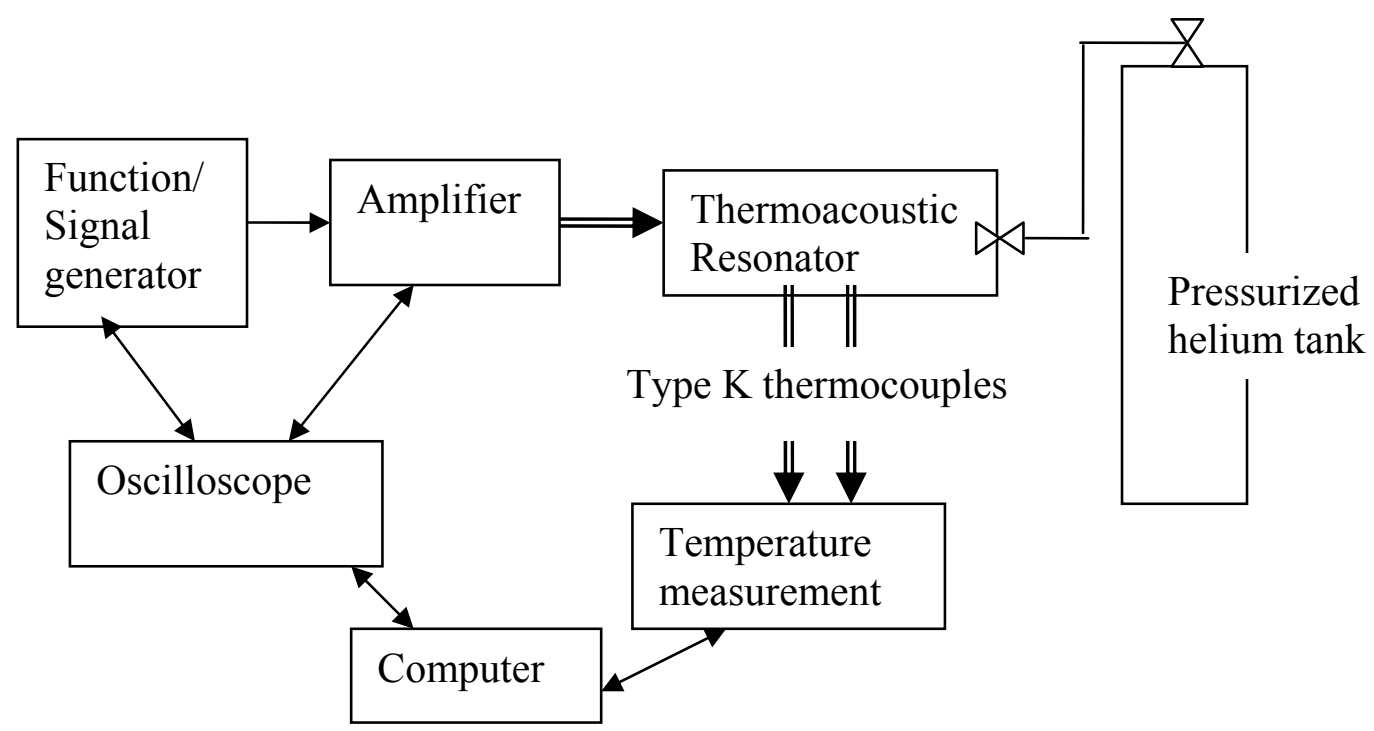

Fig. 5 Experimental set-up.

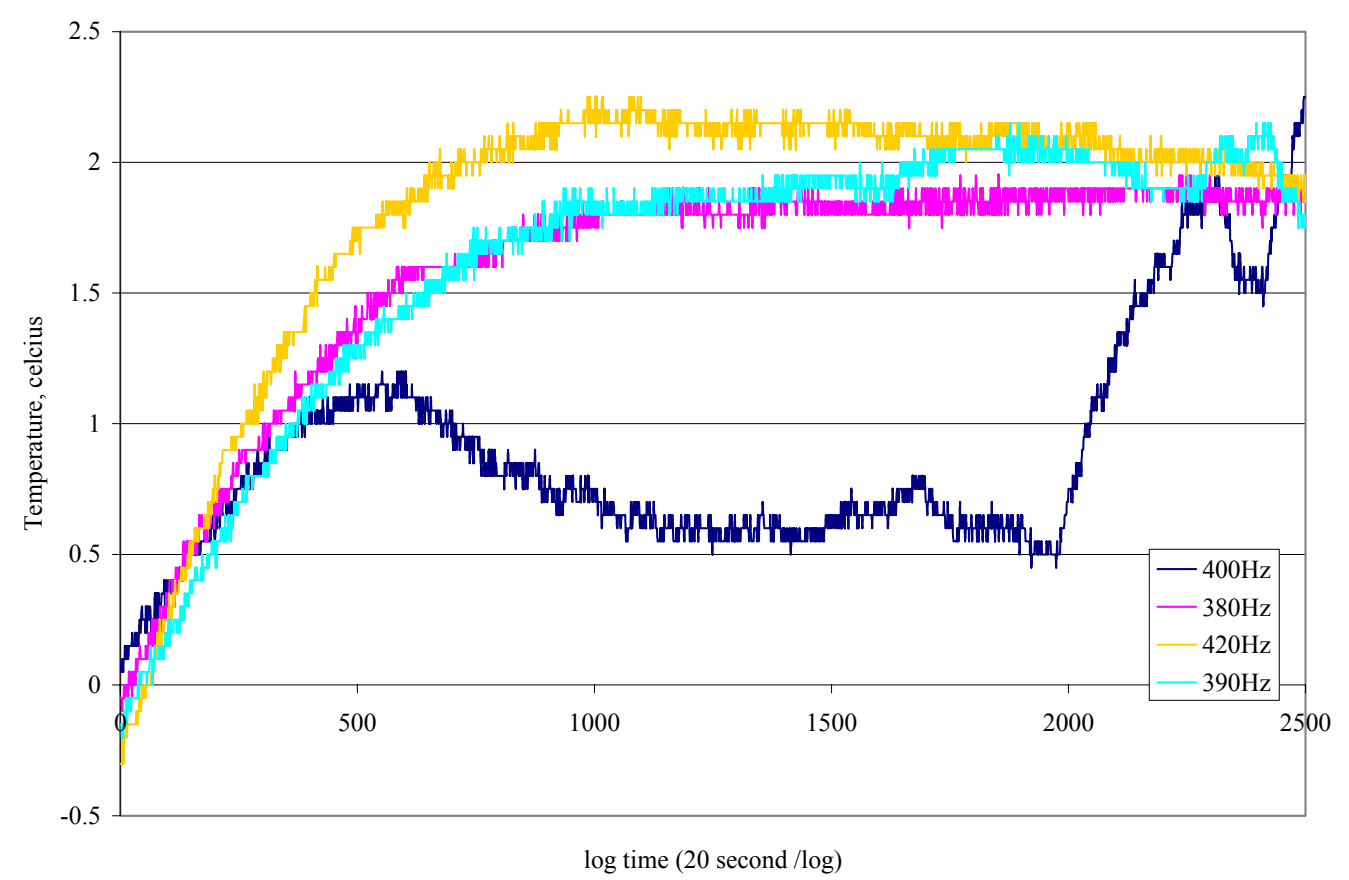

Figure 6: Temperature difference versus log time (1 bar). 


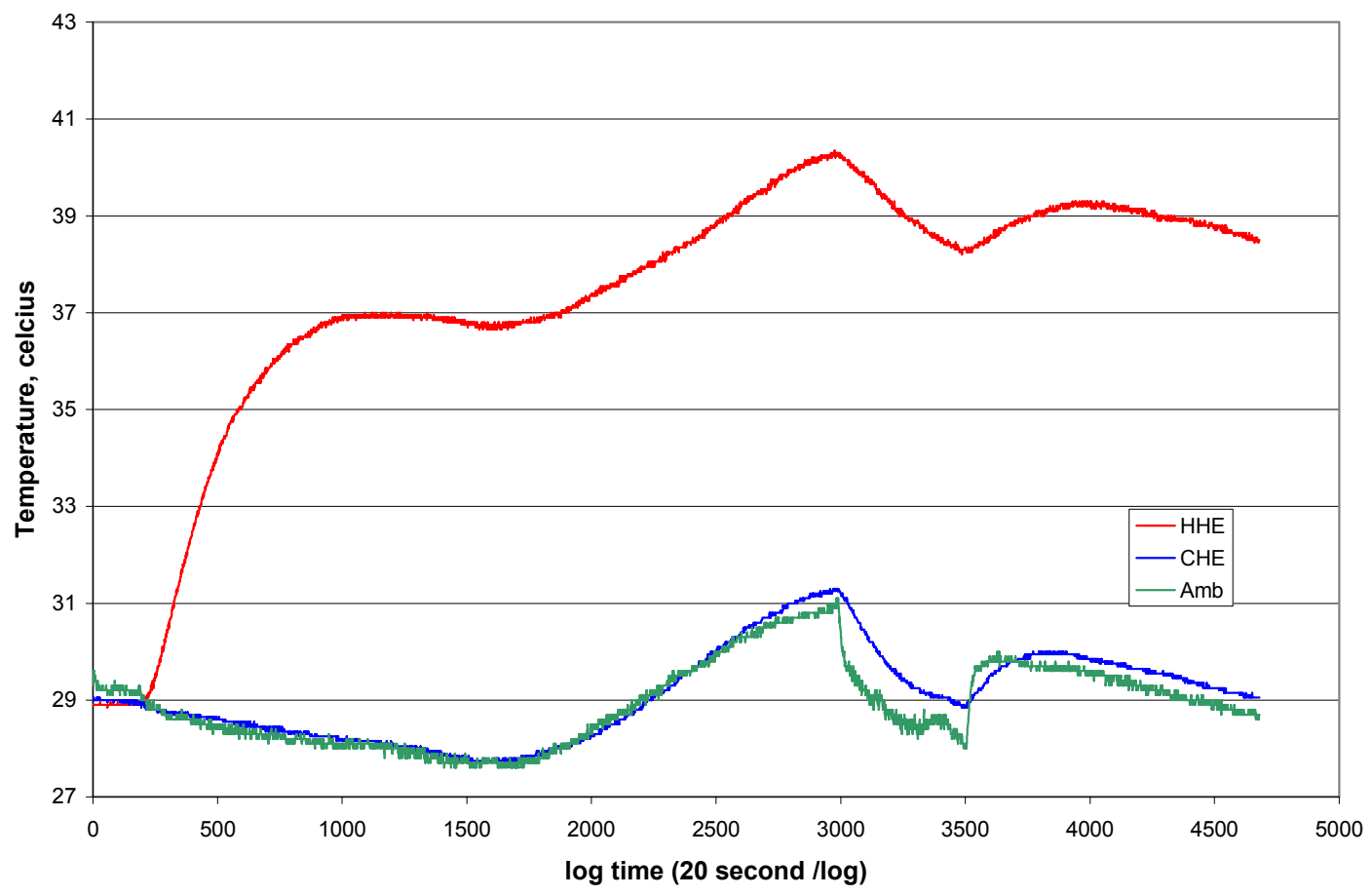

Figure 7: Temperature versus log time for $400 \mathrm{~Hz}$ ( 0.5 bar). 